\title{
Synchrophasor-based real-time state estimation and situational awareness system for power system operation
}

\author{
Heng CHEN $^{1}$, Lin ZHANG ${ }^{1}$, Jianzhong MO ${ }^{1}$, Kenneth E. MARTIN ${ }^{1}$
}

Abstract State estimation is a critical functionality of energy management system (EMS) to provide power system states in real-time operations. However, problems such as failure to converge, prone to failure during contingencies, and biased estimates while system is under stressed condition occur so that state estimation results may not be reliable. The unreliable results further impact downstream network and market applications, such as contingency analysis, voltage stability analysis, transient stability analysis, system alarming, and unit commitment. Thus, operators may lose the awareness of system condition in EMS. This paper proposes a fully independent and one-of-a-kind system by integrating linear state estimator into situational awareness applications based on real-time synchrophasor data. With guaranteed and accurate state estimation solution and advanced real-time data analytic and monitoring functionalities, the system is capable of assisting operators to assess and diagnose current system conditions for proactive and necessary corrective actions. The architecture, building components, and implementation of the proposed system are

CrossCheck date: 5 June 2016

Received: 2 December 2015/Accepted: 5 June 2016/Published online: 13 July 2016

(c) The Author(s) 2016. This article is published with open access at Springerlink.com

$\triangle$ Heng CHEN

chen@electricpowergroup.com

Lin ZHANG

zhang@electricpowergroup.com

Jianzhong MO

mo@electricpowergroup.com

Kenneth E. MARTIN

martin@electricpowergroup.com

1 Electric Power Group, Pasadena, CA 91101, USA explored in detail. Two case studies with simulated data from the subsystems of Electric Reliability Council of Texas (ERCOT) and Los Angeles Department of Water and Power (LADWP) are presented. The test results show the effectiveness and reliability of the system, and its value for realtime power system operations.

Keywords Synchrophasor, Linear state estimator, Situational awareness, Power system operation

\section{Introduction}

State estimator is one of the key applications in energy management system (EMS). It serves as a vital module that validates the raw measurements from the supervisory control and data acquisition (SCADA) system and provides current system states for downstream applications in EMS [1-3]. Applications such as contingency analysis, voltage stability analysis, transient stability analysis, system alarming, unit commitment, are critical to provide power system operators situational awareness and useful control applications to manage the grid reliability. During a system event, operators need to monitor system vulnerability, diagnose the cause of event and take corrective actions based on such tools. Typically, iterative methods are used in a conventional state estimator, which uses the previous operating condition as the initial point for the next solution. However, a state estimator may not converge, especially during the stressed condition or system experiencing contingencies when the state estimator results are needed the most. Moreover, incorrect or biased estimates are more likely to occur when the system is overloading. As a result, even when the state estimator converges, erroneous assessment of the system state may be obtained [4]. 
Some research has been conducted to increase the robustness of the state estimator by either introducing phasor measurement unit (PMU) data to formulate a hybrid state estimator or proposing new algorithms [5-10]. However, these methods are still not ready to be adopted at the control center. Authors in [11-13] have formulated the conventional state estimator problem as the linear weighed least square (WLS) problem or the mixed-integer programming problem. Nevertheless, such state estimators have not been integrated and implemented in a real power system, and it will have high computational cost to implement in large-scale power systems [14]. As a common practice in utilities for critical infrastructures, one or more backup EMSs are typically deployed. This enhances the availability of the state estimator solution, but since they may share the same data communication links and use the same solving technique, they are prone to common mode failure. A state estimator that is independent of SCADA/EMS and can be reliably solved is needed to complement the conventional state estimator.

Recent industry deployment of PMUs enables new state estimator algorithms to be developed. The synchrophasor concept was first presented in 1983 by J. S. Thorp, A. G. Phadke, and M. A. Adamiak [15]. PMU data are derived from waveform samples, time tagged using a global positioning system (GPS) clock, and typically transmitted from local substations or generating stations to control centers at 20 to 60 frames per second [16-20]. With the complex voltage and current phasor, measurement equations can be written as linear functions of the system states. The linearity between the phasor measurements and the system states allows a linear state estimator (LSE) to become feasible [21, 22].

A three phase LSE, using an algorithm developed at the Virginia Tech, was initially adopted by Dominion Virginia Power. It has been running in the field since 2013 [23]. However, this LSE is not tightly integrated with the existing real-time operation model; instead it uses a separate representation format of power system defined by the developer. It also has difficulty providing good estimate results when it encounters the system topology error. A two-level LSE [24, 25] has been developed at Washington State University (WSU). It was adopted and deployed at Pacific Gas and Electric Company [26-28]. This LSE has an advantage of detecting topology errors by running LSE at the substation level. However, this feature requires having current measurement at each of circuit breakers in a substation, which is not realistic with the present coverage of PMU installation. A three phase state estimator using modal decoupling is proposed in [14]. In order to make the least absolute value (LAV) estimator robust to leveraged measurements, system model may need to be modified with virtual buses. This is not favorable in industry practice for easy model integration. Due to the type and location of leveraged measurements, they may move the estimates towards wrong value when they carry bad data. Moreover, simulation shows such estimator takes $3 \mathrm{~s}$ to $5 \mathrm{~s}$ to solve, which cannot keep up with the real-time PMU data rate.

A reliable LSE result can provide a base case and data suitable for other synchrophasor applications, especially those aimed at wide area situational awareness. Currently, real-time operations within utilities and Independent System Operators (ISOs) rely on a SCADA system. SCADA data is generated locally at a low sample rate (one sample per 2-4 s), and most is not time-synchronized. Due to these limitations, operators do not have the ability to monitor system dynamics or have full visibility across the entire interconnected grid. Lack of such wide area visibility has been a major contributing factor to many major power system blackouts [29-31]. Power systems have also become so inter-dependent that the events in one area can quickly cascade and impact other areas. Thus, it is important to utilize timesynchronized data analytical tools to monitor wide area system stability and improve reliability.

In this paper, an integrated system of enhanced LSE and advanced synchrophasor applications for situational awareness is proposed. The system provides a fast real-time and guaranteed state estimation solution at the PMU data rate using the real power system network model. Coupled with a synchrophasor analytic applications engine and real-time visualization, the system provides dynamic visibility of the grid by a complete solution of data validation and conditioning, linear state estimation, data analytics, and visualization. Compared with the conventional state estimator, the proposed system guarantees solutions and performs in real time at the PMU data rate. By leveraging modeless algorithms and the LSE technology, the new situational awareness system extends visibility and reliable information for real-time power system operations.

The system architecture is explored in Sect. 2. Section 3 describes a data preconditioning module prior to the LSE. The system model and model update process is introduced in Sect. 4. In the following Sect. 5, an enhanced LSE handling practical issues is presented. The key synchrophasor applications engine embedded in this system is discussed in Sect. 6. Section 7 presents the one-line diagram and situational awareness visualization. The testing cases with simulated PMU data are covered in Sect. 8. The conclusion is drawn in Sect. 9.

\section{System architecture}

With the dedicated synchrophasor measurements, communication and system model topology update, the entire system can be designed to be independent of the 


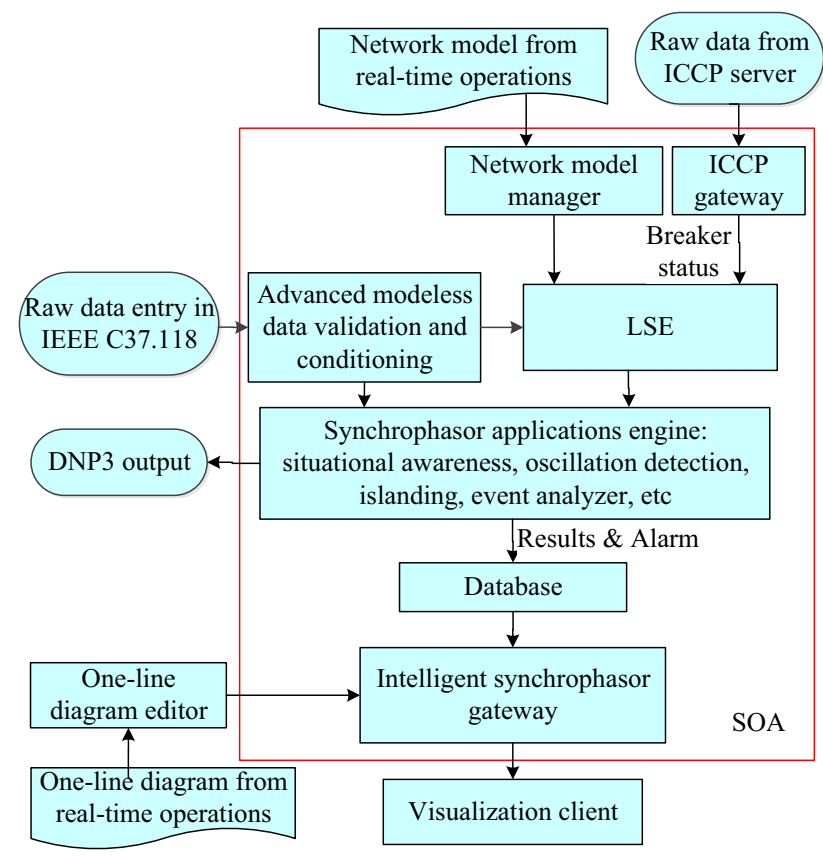

Fig. 1 Service-oriented architecture and data flow of the system

conventional EMS/SCADA system. This synchrophasorbased state estimation and situational awareness system is designed as service-oriented architecture (SOA). Figure 1 shows the architecture, building components, and data flow of the system. Synchrophasor data are transferred via high speed network to the enterprise operations service network, where different applications components are running, including modeless data validation and conditioning, network model manager, LSE, synchrophasor applications engine, etc. This extendible architecture allows integration of future synchrophasor-based applications such as contingency analysis, frequency response, protection and control. Data transfer between applications is through standard protocols, such as IEEE C37.118, Inter-control Center Communications Protocol (ICCP), and Distributed Network Protocol (DNP3). The key components of the system are discussed in the following sections.

\section{Preconditioning synchrophasor data}

PMU data quality issues do exist, especially at the early stage of PMU deployments. Hence, a preconditioning process of the raw PMU data is designed and implemented. This approach can benefit the downstream LSE by filtering out those measurements which do not meet some basic reasonability requirement. It reduces the impact of bad data on the WLS algorithm for solving the state estimation problem.

This modeless synchrophasor data validation and conditioning process contains several modules, and they process the data in a successive sequence, as shown in Fig. 2. Each algorithm examines particular characteristics of the data and records the outcome in an 8-bit quality flag.

The Communication \& Message Format module examines communication errors, protocol implementation, and message characteristics such as message length, type, and destination. If there is an error up to this point, the data is declared bad and no further checking is necessary. The Time \& Timing module checks data consistency, data time and timing error flags from the PMU, and make sure the data is

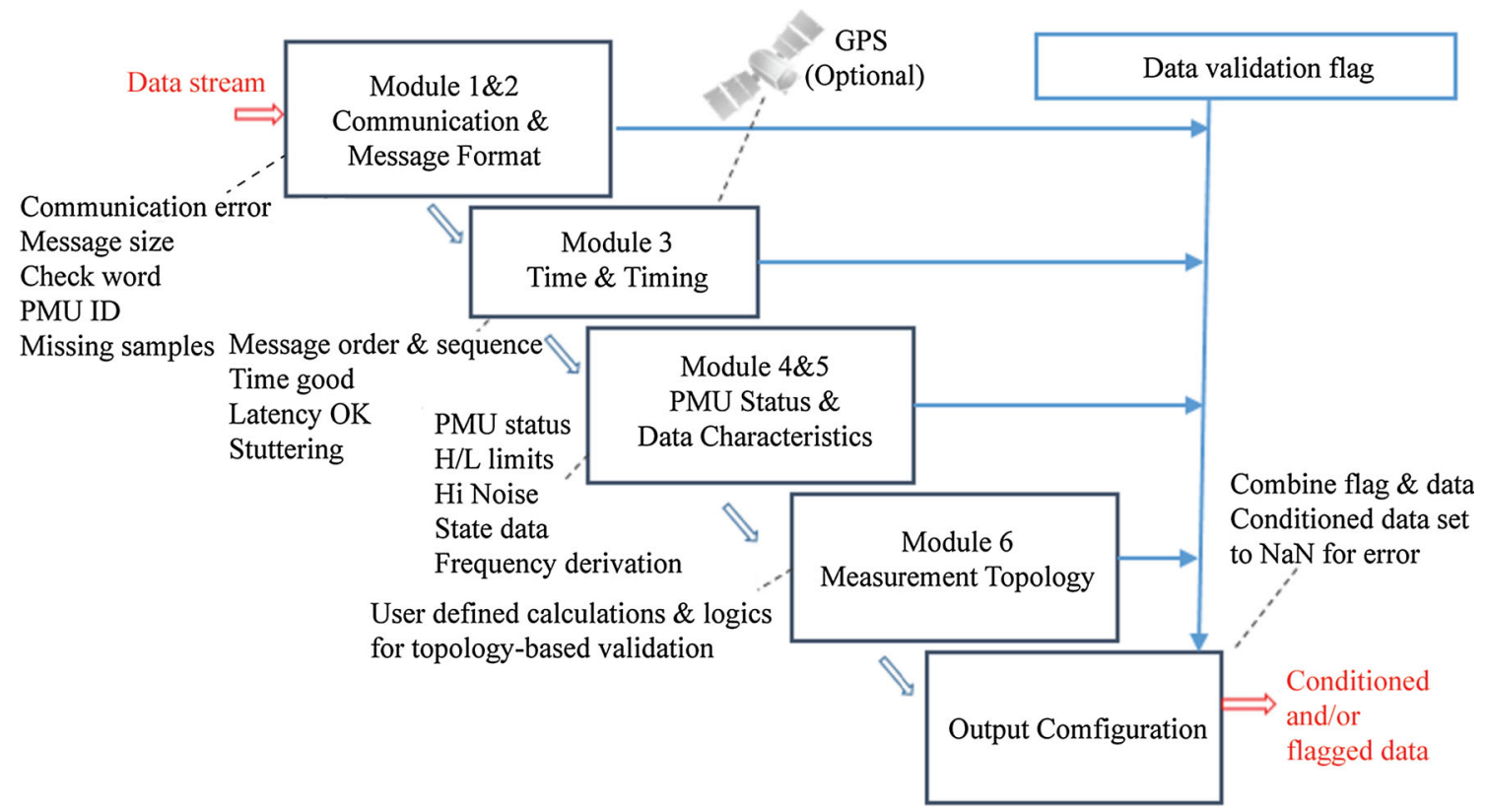

Fig. 2 Flow chart of the modeless module 


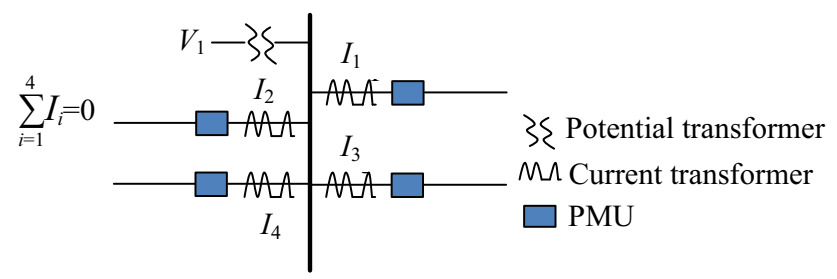

Fig. 3 KCL based topology checking for current measurements

within required latency. For example, if the timestamp in coordinated universal time (UTC) of current sample is smaller than that of previous sample, the current sample is out of order. The PMU Status \& Data Characteristics module validates data at the measurement level utilizing PMU status and measurement limit based detection. The Measurement Topology module takes consideration of topology to check the reasonability of the PMU measurements. For example, a Kirchhoff's current law (KCL) based topology check can be applied to validate the current measurements where the substation has full observability of the current phasor measurements, as shown in Fig. 3. In practice, a small tolerance $\varepsilon$, for example $10 \mathrm{~A}$, is used instead of $0 \mathrm{~A}$ in a KCL equation, since the measurements may have a small error. Current measurements that pass this topology check are used to validate the breaker statuses in the model-based LSE module. Further detail is given in Sect. 4.

\section{Network model and topology update}

Power system state estimation plays a vital role in EMS at present control centers. To solve state estimation, it requires a network model and real-time analog and digital measurements. Conventional state estimator uses SCADA data to solve the WLS problem. However, it may suffer the convergence issue when the system is under the stressed condition and the Jacobian matrix becomes singular.

With the advent of the PMU which directly measures the phasor values, a LSE becomes feasible. Linear state estimation operates in a similar way, but instead, the analog measurements are voltage and current phasors. The network model used is not necessarily the full system, because the LSE can solve pocket islands wherever the system has PMU coverage [28]. A static network model can be read once by LSE and the topology can be updated in real time via breaker status. Figure 4 shows the work flow of the network model and topology update process.

\subsection{Network model generation}

A Network Model Manager tool is developed as a module of this system. It generates the LSE XML model

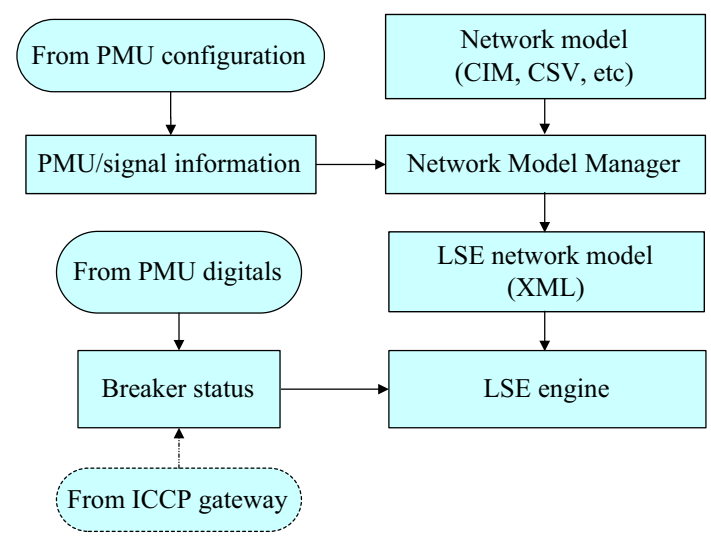

Fig. 4 Work flow of the network model and topology update process

[32] directly from a standard model used in control centers, such as the EMS node-breaker model in the common information model (CIM) format [33]. The Network Model Manager can automatically parse CIM model and integrate the required information into LSE, including substation, node, breaker, switch, transformer, line segment, series capacitor, shunt device, etc.

Regular update to EMS model is a common practice in real-time operations at ISOs and utilities. To fully leverage the updated EMS model, a model merge function is developed as a part of the Network Model Manager to allow adding network information from a new CIM model to an existing LSE model. The attributes from the new CIM model take precedence over those from the previous network model, and the network model is updated accordingly. For example, if the impedance of a transmission line segment in the new CIM model is different from the existing LSE network model, the transmission line is updated with parameters from the new CIM model. However, user can prevent the Network Model Manager from overwriting the elements in the model that are user defined. All model changes are merged automatically by the program and the changes are highlighted using different color codes. Through the Network Model Manager, the LSE is well integrated with existing up-to-date real-time operation model used at ISOs and utilities.

\subsection{Real-time model topology update}

The LSE is designed and implemented to obtain the breaker status from PMU data, and alternatively from EMS/SCADA through an ICCP gateway. Digital statuses from PMU or object points from ICCP are mapped to the corresponding breakers in the model.

In the case of using ICCP for breaker status update, SCADA data reporting is $2 \sim 6 \mathrm{~s}$ delayed from the PMU data. During this time interval, the LSE may have a 
mismatch between measurement and model, which can create significant solution errors. Moreover, some breaker statuses may be reported incorrectly. Therefore, a function is developed in this LSE to validate and update the breaker status in real time, based on the PMU current measurement topology check discussed in the preconditioning module.

For each data frame, LSE uses current measurements to validate and correct the corresponding breaker status of the line. If the current magnitude is less than a very small value $\varepsilon$, the statuses of the associated two breakers (assuming this is a breaker-and-a-half scheme) should be open. If the breaker statuses are not open according to SCADA, LSE can be configured to overwrite the breaker statuses with inferred values. Thus, the topology error can be corrected by LSE.

Figure 5 shows a typical breaker-and-a-half scheme substation configuration, which is widely deployed in $500 \mathrm{kV}$ level systems. It has the full observability with PMU current measurements. Thus, the KCL topology check can be applied in the preconditioning module to validate all the current measurements. And the LSE can use the validated current measurements to infer breaker statuses. For example, in field practice, if the magnitude of current $\mathrm{I} 3$ is less than $10 \mathrm{~A}$, the breakers $\mathrm{F}$ and $\mathrm{G}$ should be open.

\section{LSE algorithm}

The key component of the system is a LSE. LSE has four core modules: topology process, observability analysis, state estimation, and bad data detection. Figure 6 shows the basic algorithm and data flow of the LSE.

The function of the network topology processor is to determine the present topology of the network from the

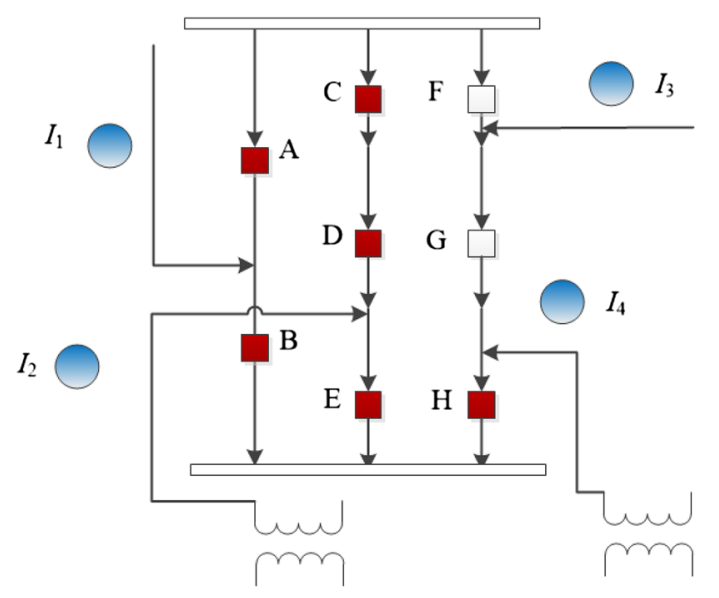

Breaker closed; $\square$ Breaker open;

PMU current measurement

Fig. 5 Use of PMU current measurements to infer breaker status

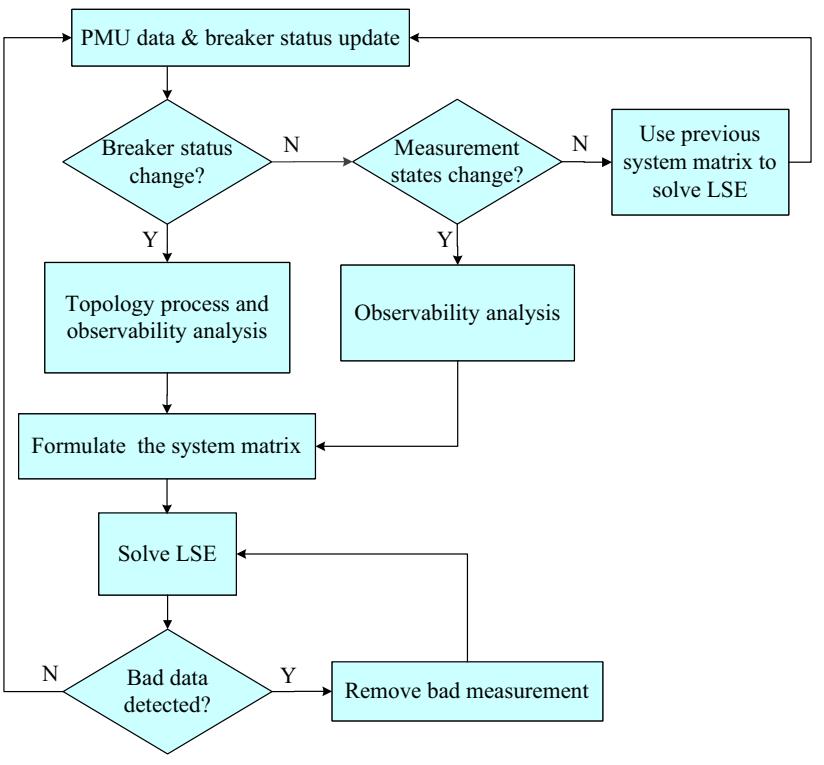

Fig. 6 Flow chart of LSE algorithm

telemetered status of circuit breakers. As the topology of the system changes in real time, a real-time observability analysis is required to correlate the PMU measurements with the topology, so that the LSE is able to identify observable islands. The LSE problem can be formulated and solved in these islands individually, without creating equivalence of the rest of the system. Observability analysis can identify special cases. For example, if the bus in a substation is split into two topological buses, one may be observable because it is directly measured by a PMU or indirectly measured by a PMU at the other end of a line, whereas the other bus may not have a direct or indirect measurement so it is not observable. The LSE code is enhanced so it can recognize split busses and update observability issues. Observable voltage and current phasors in addition to those directly measured by a PMU can be identified and included in the LSE output stream. This function extends the PMU coverage of the power system, and provides more insights of grid dynamics for real-time operations.

The state estimation problem is then formulated and solved. The LSE solves each observable island separately. The fundamental LSE algorithm is well explored in $[23,25,27]$. The formulation of linear state estimation problem is described as follows,

$\begin{cases}\min & \boldsymbol{\sigma}^{\mathrm{T}} \boldsymbol{W} \boldsymbol{\sigma} \\ \text { s.t. } & \boldsymbol{Z}=\left[\begin{array}{l}\boldsymbol{I} \\ \boldsymbol{Y}\end{array}\right] \boldsymbol{X}+\boldsymbol{\sigma}\end{cases}$

where $\boldsymbol{Z}$ is the measurement vector including complex bus voltage and line current phasors; $\boldsymbol{X}$ is the state vector which is related with the measurement vector by the system matrix; $\boldsymbol{I}$ is an identity matrix; $\boldsymbol{Y}$ stands for the relationship 
between voltage and current; $\boldsymbol{\sigma}$ is a vector of the error in each of the measurements; and $\boldsymbol{W}$ is the covariance matrix of measurement errors.

WLS method is widely used in conventional state estimator which has been deployed in most of the EMS at ISOs and utilities. The robustness of the method meets the realtime operation requirements. Given the more accurate PMU data [34] and various testing, the WLS with bad data identification for LSE is sufficient for practical real-time control center application. The robustness will be further improved by the extended PMU coverage as the industry deploying more PMUs in the field.

Under normal condition, if there is no change of breaker status or measurement state, LSE uses the previous system matrix. The execution time is very short, around $7 \mathrm{~ms}$ for the size of a 3091 node system, and the LSE can keep up with the regular PMU data rate (30 or 60 samples per second). Hence, LSE is able to track the system transients and validate the PMU data under transient conditions. A measurement state change means that the measurement value changes from a valid number to Not a Number $(\mathrm{NaN})$ or vice versa. This condition usually occurs when the PMU measurement is delayed or dropped out from the upstream phasor data concentrator (PDC). In this case where only measurement state changes but not the topology of the system, LSE only needs to re-conduct the observability analysis and reformulate the system matrix that is used to solve the LSE problem. The delayed and dropped measurements are not considered as input for the LSE. Some buses may not be observable anymore. However, if the nodes of the delayed or dropped measurement are still observable through other PMU measurements, the LSE will be able to estimate and replace the missing measurements with estimated values. This also saves the execution time of topology process. The LSE takes around $50 \mathrm{~ms}$ to execute in this case. If breaker status changes, the LSE needs to run through all the modules to update the topology. Under this condition, the execution time of this particular frame is longer, around $150 \mathrm{~ms}$. However, note that the system topology does not change very often in a short period of time and neither does the measurement state. When these changes occur, the LSE output falls a little behind the measurement stream, but since the normal execution speed is about twice the reporting rate of 60 samples per second, it can quickly catch up. The LSE can hence operate properly in real time at the PMU data rate.

A bad data detection module is run on a solution set to detect and eliminate bad measurements. The usual Chisquare test is implemented in the LSE to detect bad data, as it is widely used in production-grade conventional state estimators. If the measurement residual vector is $\hat{e}$, the weighted sum of its square $\hat{f}$ is a random variable which has a Chi-square probability distribution [35], i.e.

$\hat{f}=\sum_{j=1}^{N_{\mathrm{m}}} \frac{\hat{e}_{j}^{2}}{\sigma_{j}^{2}} \quad j=1,2, \ldots, N_{\mathrm{m}}$

where $N_{\mathrm{m}}$ is the number of measurements; $j$ is the measurement index; and $\sigma_{j}$ is the covariance factor of the error.

The expected value of $\hat{f}$ is equal to the number of degrees of freedom, and this can be used to determine whether bad data is present or not. With the preconditioning module discussed in Sect. 3, the Chi-square test method for bad data detection is practical and sufficient for the proposed system. Identification of the bad data also follows standard procedures used in the conventional state estimators. Once bad data is identified, the state estimation is reformulated with the bad data removed, and solved again until there is no bad data present.

\section{Synchrophasor applications engine}

The other core part of the system is a synchrophasor applications engine. This engine platform performs calculations and alarm/event detection, and acts as a data hub for synchrophasor applications. It also connects a database, which stores raw synchrophasor data with full resolution, LSE estimated values, user-defined calculation values, data processing results, as well as alarms and events. An application programming interface (API), called the intelligent synchrophasor gateway (ISG), provides access to the application platform and the archived data in the database. This API provides easy access for third party or customized applications, and also simplifies integration of new synchrophasor applications into the system. Data processing in all modules of the system use the full resolution of the PMU data and LSE results, and the applications operate fast enough with a data rate up to 60 samples per second.

The LSE results provide more reliable and accurate PMU data to synchrophasor applications for wide-area situational awareness, real-time dynamics monitoring, as well as event analysis of the power grid. Typical power system applications [36-39] are integrated in this system, such as:

1) Wide Area Situational Awareness

2) Phase Angle and Grid Stress Monitoring

3) Voltage and Angle Stability Analysis

4) Ambient Oscillation Analysis

5) Oscillation Detection

6) Islanding Detection

7) Generation and Load Trip Detection 
8) Automated Event Analyzer

9) Intelligent Alarming

These applications run in parallel at the PMU data speed, which helps improve operators' visibility to system dynamics and events, and assists operators to take actions to improve system reliability. For instance, the Automated Event Analyzer application provides grid operators with information immediately following a system event. It continuously performs disturbance detection, disturbance classification, and disturbance location estimation in real time, including oscillations, voltage and angle stability, islanding, generation and load trip, line trip, and composite alarm conditions. It presents a summary of events including all the relevant information to users via a yellow pop-up dialog box. The pop-up dialog box summarizes the event type, name, and occurrence time, and it appears in the approximate location of the event on the geospatial map. Figure $7 \mathrm{a}$ is a screenshot of the system application dashboard, including a geospatial map with measurement layers on the left, two line charts showing system frequency and power flow value, an alarm view in the upper right corner, and two system mode damping views in the bottom right corner. When a generation trip event occurs, the Automated Event Analyzer application correctly detects the event, and pops up the yellow dialog box close to the event location on the geospatial map in Fig. 7a. By clicking on the "more" button on the event summary shown in Fig. 7b, it can bring up detailed diagnostics showing all of the relevant metrics to operators at a glance. Figure $7 \mathrm{c}$ shows the detailed metrics visualization of this generator trip event. The metrics in the screenshot are, from upper left to bottom right, system frequency, area control error (ACE), power flow, voltage magnitude trend, mode trend, key voltage profile, voltage sensitivity, and oscillation damping ratio.

\section{Situational awareness and one-line diagram visualization}

Situational awareness could be described as fully informed of the present condition and state of the power system and all other entities that could have an effect on it. This first includes the power system itself and the state of all its elements including buses, lines, switches, generators, transformers, etc. Secondly it includes conditions and entities that could have an effect on the power system, such as severe weather, fires, earthquakes, social disturbances and events that cause large load changes. Situational awareness requires bringing these issues to the attention of the operator to the greatest extent possible.

A visualization client provides the measured and derived power system states, and analytical results information to

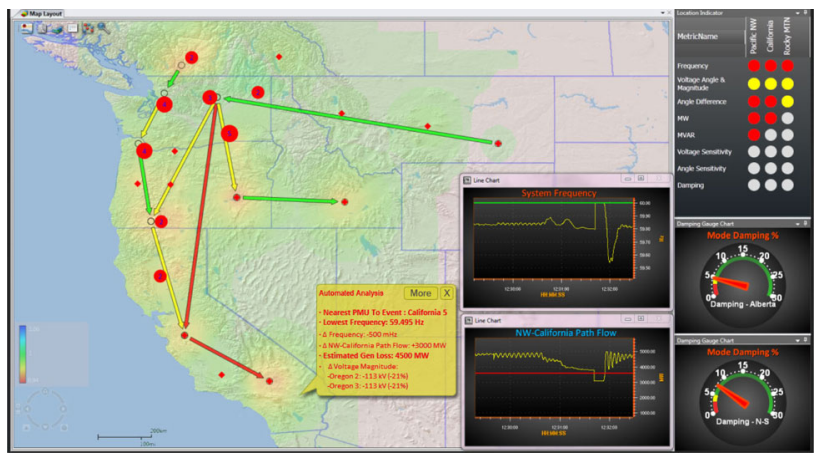

(a) Automated Event Analyzer application on dashboard

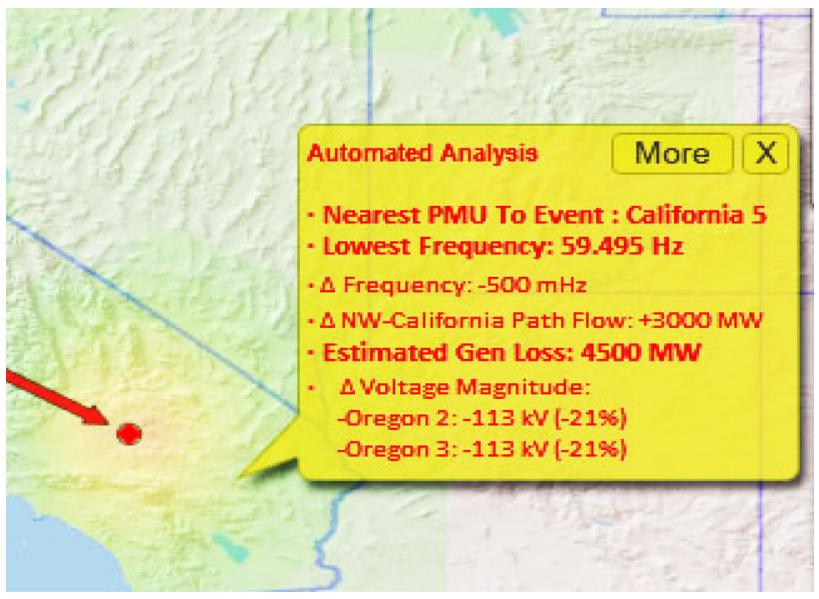

(b) Pop-up dialog box of the event summary

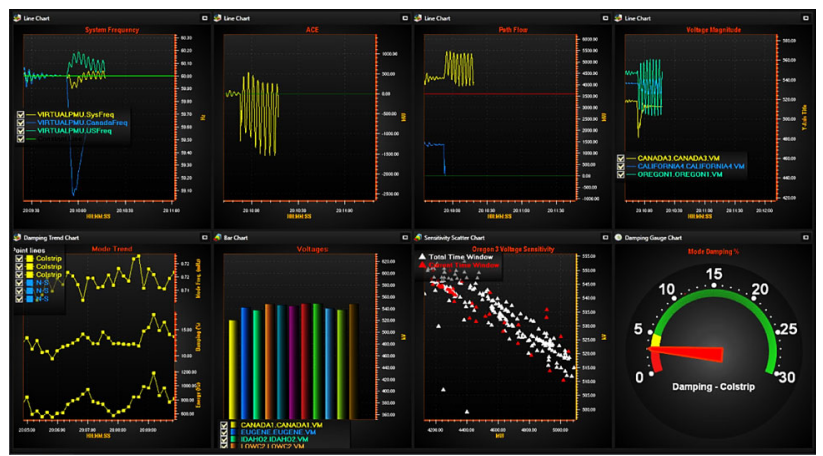

(c) Automated Event Analyzer with relevant detailed metrics

Fig. 7 Screenshots of Automated Event Analyzer application

users. The client has the capability of providing not only power system data, but also none measurement information to the users, such as traffic, weather, and fire layers on a geospatial map. The visualization is a composite of individual displays from different information sources which are selected by the user. The user can save the selected display set as a profile, and multiple profiles can be saved for different users or user groups.

The visualization client is composed of many modules, including charts, maps, layers, alarms, events, etc. These 


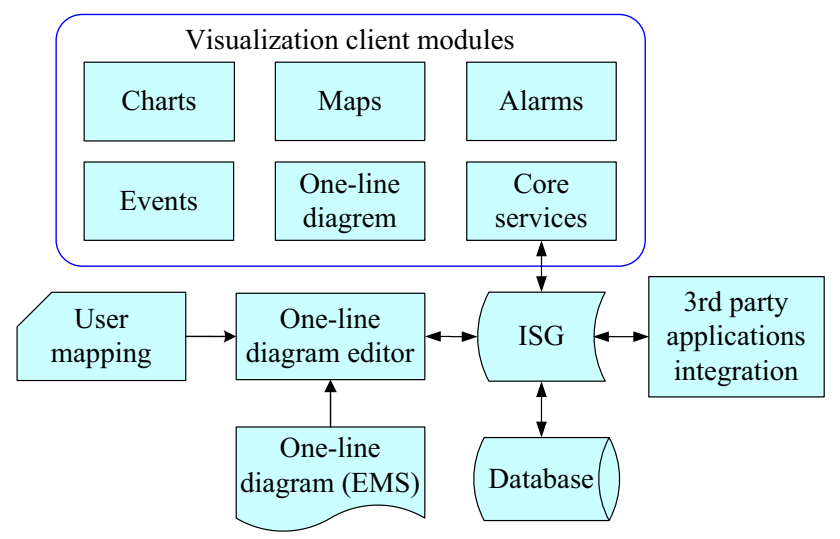

Fig. 8 Block diagram of the visualization client

visualization modules are developed by using the advanced technology, which supports data refresh rate up to 120 frames per second. It provides situational awareness of true power system dynamics without downgrading any resolution of the data. Figure 8 shows the block diagram of the visualization client. The functionality of each module is independent. This design makes the system flexible enough to accommodate new functionality, or change the existing functionality in future without affecting other modules.

Power system engineers and operators are familiar with one-line diagrams. In order to have a seamless transition for users to use the proposed system, a one-line diagram module is also developed. The one-line diagram has two levels of visualization. The top level is the system wide view. Bulk power system transmission lines, substations and their connectivity are displayed in this level of view. From system wide view, user can drill down to the substation-level visualization, where bus, breaker, switch, transformer and line configuration are displayed.
Interactions between visualization modules are made available for easy information correlation. For example, Fig. 9 shows a screenshot of the system application display, including a geospatial map and an one-line diagram of a substation. A trend chart view showing a bus frequency is popped up from the one-line diagram.

For easy maintenance and support, it is also preferred to leverage existing one-line diagrams from users' current EMS. The system provides the capability to import oneline diagrams through the one-line diagram editor. User can also create, edit and update one-line diagram files, as well as map both PMU measurements and LSE estimated results on the electrical components in the one-line diagram. User can define dynamic behaviors to power system shapes of the one-line diagram by setting logic conditions. For instance, change of color on change of value, change of shape on change of value thresholds. One-line diagram information is stored in a database for providing centralized access for all visualization clients using ISG. These diagrams are stored by using versions for backward compatibility and change management.

In the process of a model upgrade, the one-line diagram should be upgraded correspondingly. User can import updated one-line diagrams to the system. Then the modified one-line diagrams can be saved as new versions for use. All of the configured data mapping and logical condition mapping are retained.

\section{Case studies}

The system is tested with two use cases, one with an ISO focusing on monitoring the critical part of grid as an alternate independent system, and the other case for a local

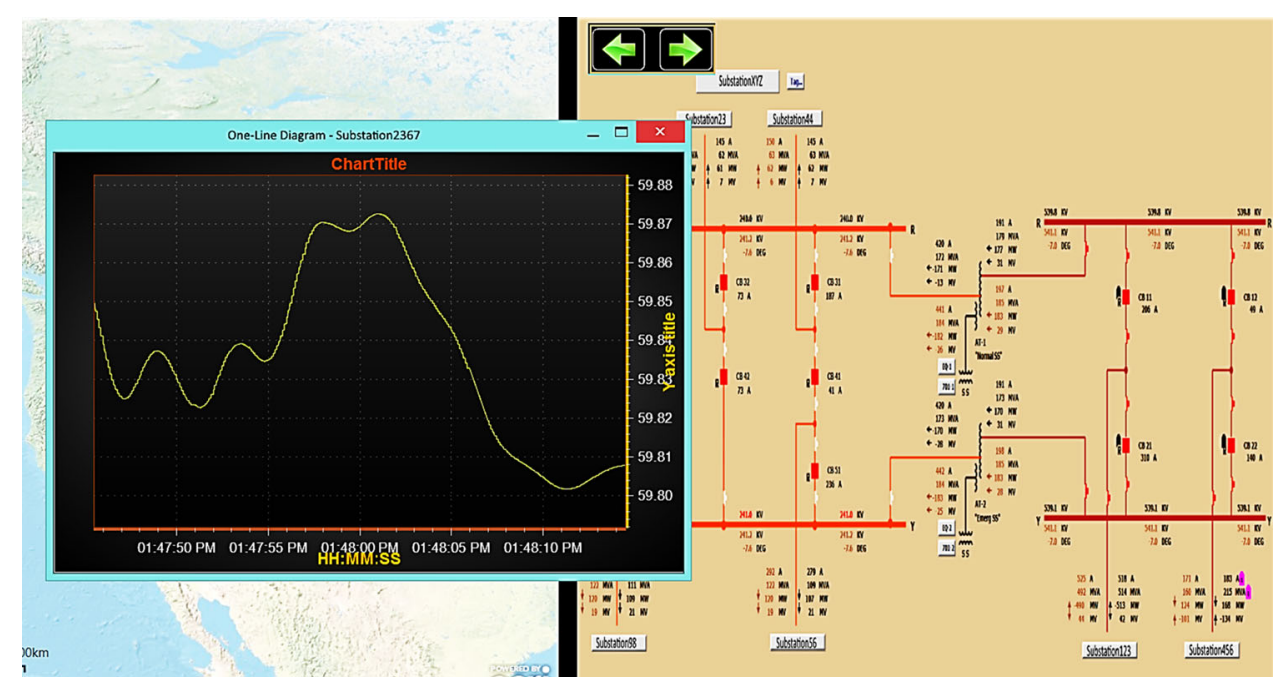

Fig. 9 Interaction between visualization modules 


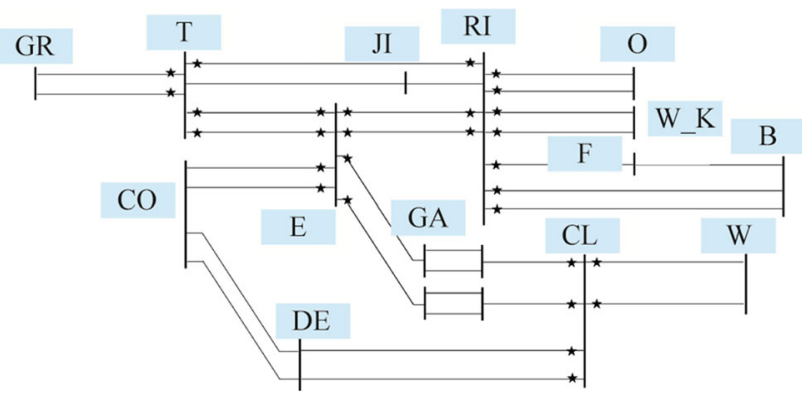

Fig. 10 ERCOT subsystem diagram

utility with a focus on extending PMU observability to assist line switching action. The detail case examples are presented as follows.

\subsection{Monitoring critical part of the grid}

A subsystem of the Electric Reliability Council of Texas (ERCOT) is modeled and implemented as a case study. As shown in Fig. 10, the subsystem consists of fourteen 345 $\mathrm{kV}$ substations which are located in the northwest region of ERCOT footprint. Model parameters and simulated ambient PMU data are obtained from a Transient Security Assessment Tool (TSAT) simulation case. Four substations out of the fourteen have simulated PMU voltage and current phasor measurements set up in the simulation. The measurement locations are marked by using "star" in Fig. 10. The simulated PMU data is streamed into the system and used as input for the LSE. LSE extends the PMU coverage to all substations and transmission lines of the subsystem, conditions the data, and streams the results into the synchrophasor applications engine for analysis.
Figure 11 shows the visualization dashboard of this subsystem, including geospatial map with voltage and angle measurements of entire ERCOT footprint, corresponding one-line diagram of the subsystem, aggregated system frequency value, frequency trend, and alarm view. Voltage magnitude and angle from LSE results are mapped beside the corresponding buses, and calculated active and reactive power flows are mapped to the corresponding transmission lines of the one-line diagram in Fig. 11. The system wide view is drawn by using the one-line diagram editor discussed in Sect. 7. Although PMU coverage is not $100 \%$, operators can still monitor the key buses and tielines of the system, and obtain a good confidence level of the current grid operating condition.

From the dashboard, user can drill down to a specific substation, where user can see the detailed node-breaker connection inside the substation. Figure 12 shows a display of substation T and E. LSE estimated voltage magnitudes are mapped to the corresponding nodes in the substation one-line diagram on the left side of the screenshot. Calculated individual transmission line power flows are trended in real time on the right side. Metrics of multiple substations can be configured in the same display for monitoring, including voltage profile, power flows, phase angles, etc. User can not only identify which substation has the PMU information but also which nodes the PMU is monitoring. This is particular useful to show if the PMU is installed on the line side or the bus side, so that operators can have a direct visualization of which measurements can still be trusted when a split bus configuration or a line outage condition occurs.

All the substation-level one-line diagrams are directly imported from existing ones used in ERCOT real-time

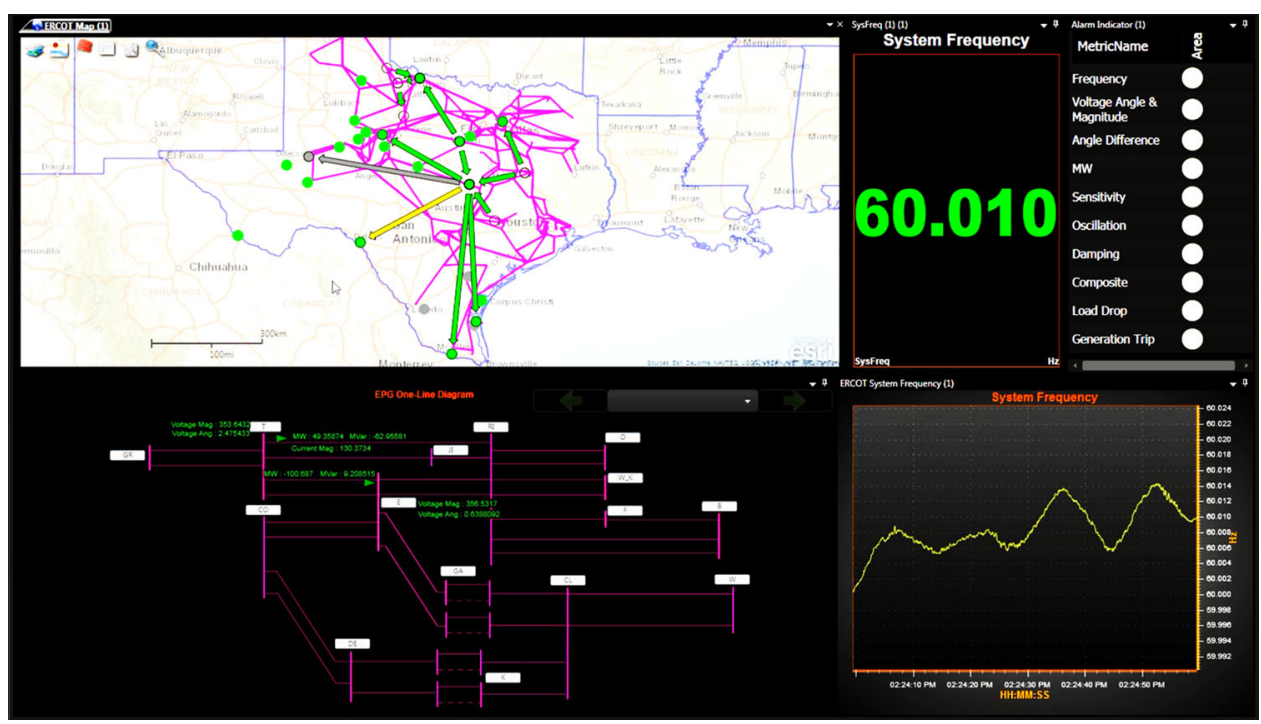

Fig. 11 Situational awareness dashboard of the ERCOT subsystem 


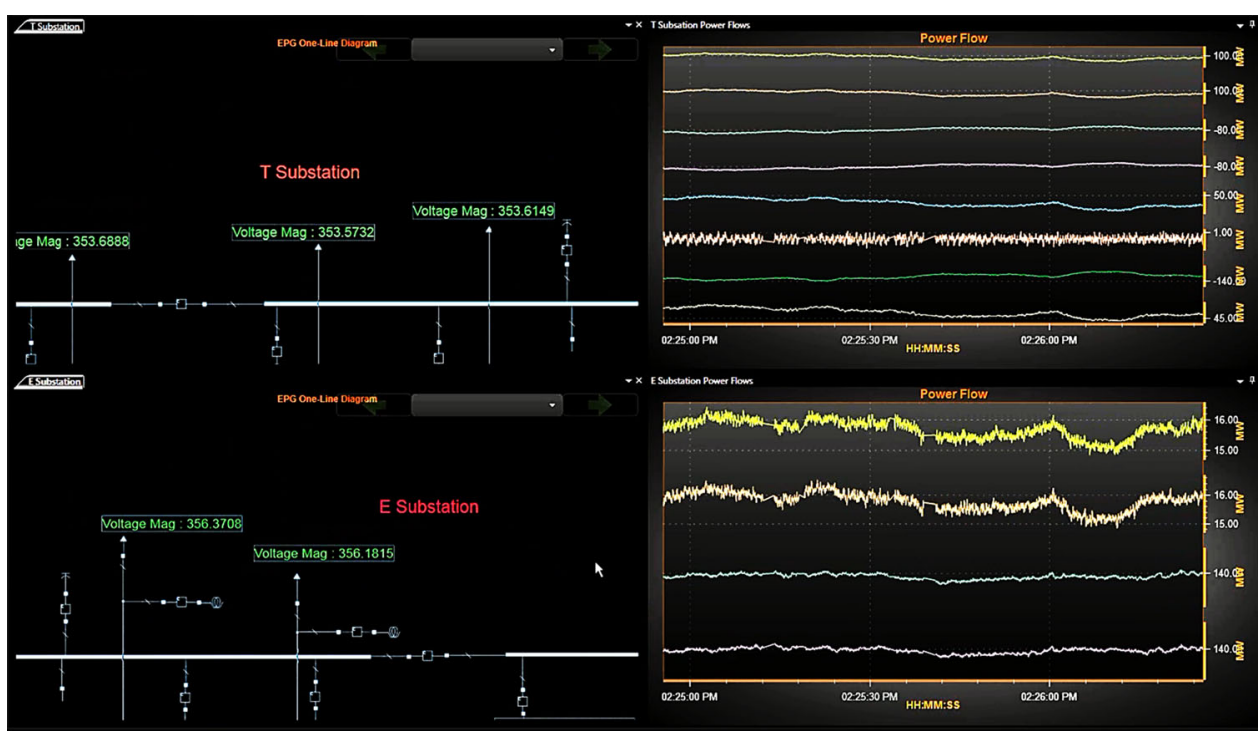

Fig. 12 Substation display with one-line diagram and data trend

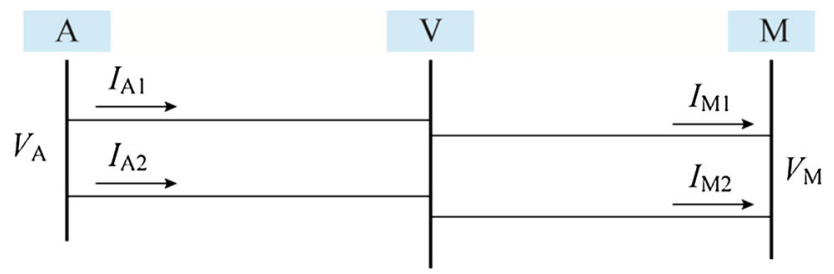

Fig. 13 LADWP subsystem diagram

operations. This integration allows leveraging existing business process within an organization without introducing additional redundant work.

With similar approach, the system is easily adapted to the entire ERCOT $345 \mathrm{kV}$ network using current real-time operation model. In case the EMS fail for any reason, this system can still provide critical state estimation solution and further feed reliable data to synchrophasor applications. Such information is valuable to assist operators in monitoring and taking corrective actions in real-time operations.

\subsection{Assisting line switching action}

A subsystem of the Los Angeles Department of Water and Power (LADWP) is modeled and implemented as another case study. The subsystem consists of three substations and four transmission lines, as shown in Fig. 13. There are two $500 \mathrm{kV}$ transmission lines connecting substation $\mathrm{V}$ and $\mathrm{A}$, and two $500 \mathrm{kV}$ transmission lines connecting substation $\mathrm{V}$ and $\mathrm{M}$.

One PMU is installed at each of the substations A and M. Each PMU measures the substation voltage and both line currents. There is no PMU installed at substation V. Currently at LADWP, the phase angle difference between substation $\mathrm{A}$ and $\mathrm{M}$ is monitored for grid stress analysis. However, when one of the two lines between substation $\mathrm{V}$ and $\mathrm{M}$ is tripped and needed to be reclosed, there is no direct measurement for phase angles between these two substations. The proposed system can be used to estimate the voltage phasor at substation $\mathrm{V}$ to directly address this issue, and provide situational awareness for operator action.

A heavy summer loading case of the Western Electricity Coordinating Council (WECC) is used in simulation to generate PMU data. The voltage and current phasor at substation $\mathrm{A}$ and $\mathrm{M}$ are simulated to mimic the field measurements. One transmission line between substation $\mathrm{V}$ and $\mathrm{M}$ is tripped at one minute into the simulation. The simulated PMU data is streamed into the system and used as input for the LSE. LSE results are then streamed into the synchrophasor applications engine for analysis.

Figure 14 shows the visualization dashboard of this subsystem, including a geospatial map with phase angle difference measurements, alarm view, and corresponding one-line diagram of the subsystem. Circuit breakers are represented on the one-line diagram by square boxes. When a breaker is closed, the icon is red and otherwise is green. Voltage magnitude and angle from LSE results are mapped beside the corresponding buses, and calculated active and reactive power flows are mapped to the corresponding transmission lines.

Right after the line trip, the power flow reroutes to the other parallel line, and the grid becomes more stressed. This results in the phase angle difference between substation $\mathrm{V}$ and $\mathrm{M}$ increasing. The proposed system detects the 


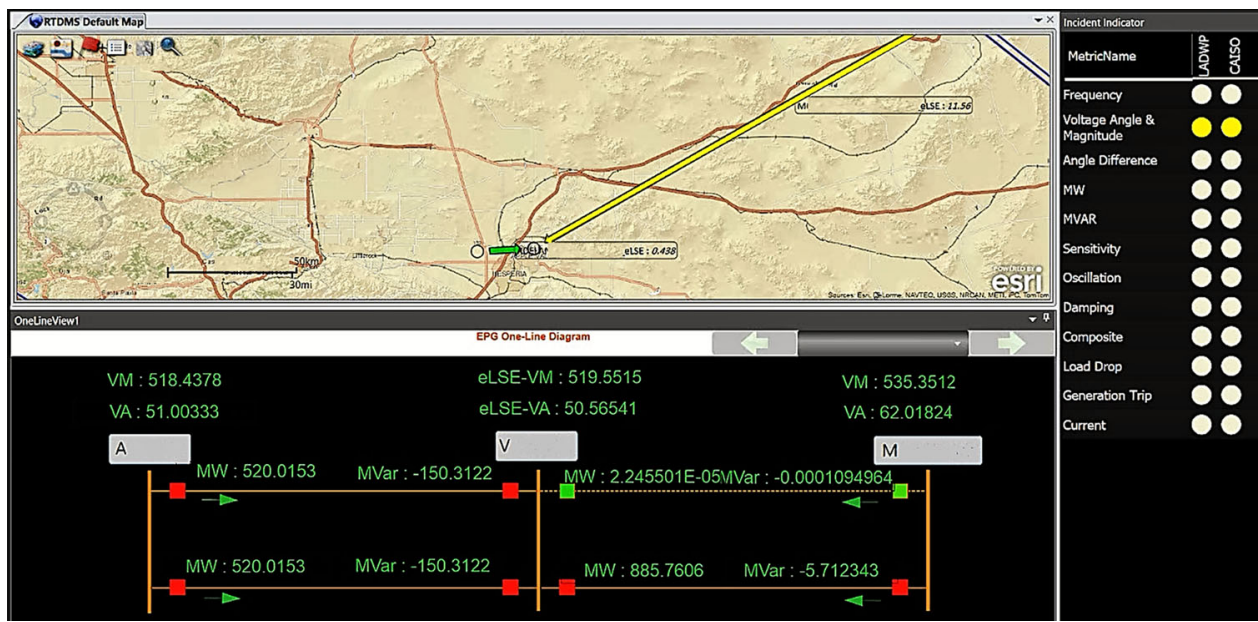

Fig. 14 Visual indication and alarm after line trip on situational awareness dashboard

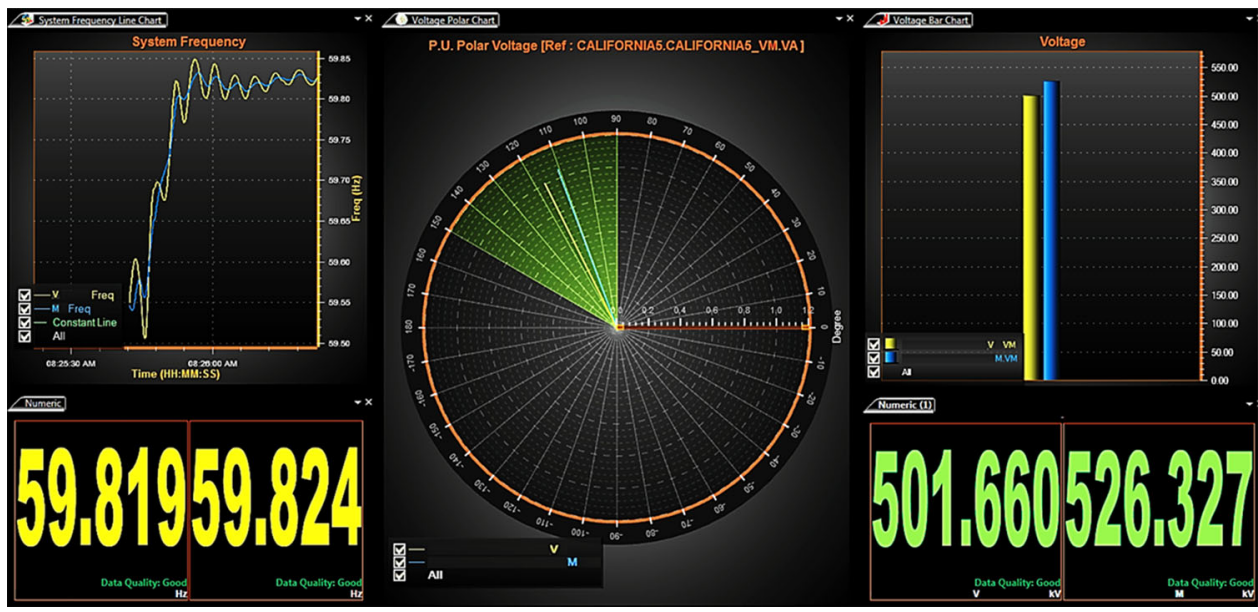

Fig. 15 Line switching assistant display

event and alerts the operator with yellow indicator in the alarm view, and with the color of the corresponding angle pair turning to yellow in the geospatial map, as shown in Fig. 14. The circuit breakers of the tripped line are also shown as open in green.

To assist line closing, a line switching assistant display is available in the system. An example is shown in Fig. 15. User can re-configure the display panel with new visualization templates and data as needed. In order to close the breaker, the differences of frequency and voltage signals from the two ends of the open transmission line need to be within thresholds respectively. In this example, trend chart, bar chart and numerical chart are used to monitor them side by side. Additionally, the phase angle difference between the two ends needs to be within the closing angle limit. A polar chart is used to visualize this information, as shown in the middle view of Fig. 15. The green segment of the polar chart is defined by the closing angle limit, which indicates the safe condition to close the line back to service. By simply using this display, operator can issue safe line switching action. Such monitoring display provides an intuitive way for operators to assess the system situation, and it also helps operators take actions in real-time operations.

\section{Conclusion}

A fully independent synchrophasor-based real-time situational awareness system with LSE is presented. The system provides the state-of-the-art capability to use timesynchronized data for operators to monitor the grid effectively, to assess current system conditions, to diagnose the situation, and to take proactive and necessary corrective actions. LSE provides guaranteed solutions and extended system visibility. It also validates and conditions PMU data 
for grid operation. The proposed system is fully developed and tested by using simulated data and live data in production. The system is proved useful for faster and more reliable real-time state estimation and synchrophasor applications for situational awareness. It is believed that with more installed PMUs, improved data quality, and enhanced LSE, the synchrophasor-based situational awareness system will provide greater visibility and dynamic insights to improve the grid stability and reliability in real-time power system operation.

The system is also designed as a comprehensive platform, which is flexible to allow adding additional modules. For future work, the authors are exploring other applications to be integrated in the proposed system, such as LSEbased real-time contingency analysis, dynamic phase angle for overloading, real-time model error detection, and transmission equipment health monitoring.

Acknowledgments The authors would like to acknowledge Bill Blevins and Sarma Nuthalapati at Electric Reliability Council of Texas (ERCOT), Tim Bradberry and Megan Vutsinas at Duke Energy, Kyle Thomas and Kevin Jones at Dominion Virginia Power, and Anthony Faris at Bonneville Power Administration (BPA) for providing valuable suggestions and feedback on design, development and testing of this work.

Open Access This article is distributed under the terms of the Creative Commons Attribution 4.0 International License (http:// creativecommons.org/licenses/by/4.0/), which permits unrestricted use, distribution, and reproduction in any medium, provided you give appropriate credit to the original author(s) and the source, provide a link to the Creative Commons license, and indicate if changes were made.

\section{References}

[1] Bose A, Clements KA (1987) Real time modeling of power networks. Proc IEEE 75(12):1607-1622

[2] Abur A, Exposito AG (2004) Power system state estimation: theory and implementation. Marcel Dekker Inc, New York

[3] Schweppe FC, Handschin EJ (1974) Static state estimation in electric power systems. Proc IEEE 62(7):972-982

[4] Tecchio PPV, Benedito RAS, Alberto LFC (2010) The behavior of WLS state estimator near the maximum loadability point of power systems. In: Proceedings of the 2010 IEEE power and energy society general meeting, Minneapolis, 25-29 Jul 2010, 6 $\mathrm{pp}$

[5] Mili L, Cheniae MG, Rousseeuw PJ (1994) Robust state estimation of electric power systems. IEEE Trans Circ Syst-I 41(5):349-358

[6] Baldick R, Clements KA, Pinjo-Dzigal A et al (1997) Implementing nonquadratic objective functions for state estimation and bad data rejection. IEEE Trans Power Syst 12(1):376-382

[7] Celik MK, Abur A (1992) A robust WLAV state estimator using transformations. IEEE Trans Power Syst 7(1):106-113

[8] Abur A, Celik MK (1991) A fast algorithm for the weighted least absolute value state estimation. IEEE Trans Power Syst $6(1): 1-8$
[9] He GY, Dong SF, Qi JJ et al (2011) Robust state estimator based on maximum normal measurement rate. IEEE Trans Power Syst 26(4):2058-2065

[10] Chen YB, Ma J, Zhang P et al (2015) Robust state estimator based on maximum exponential absolute value. IEEE Trans Smart Grid. doi:10.1109/TSG.2015.2485280

[11] Gomez-Quiles C, de la Villa Jaen A, Gomez-Exposito A (2011) A factorized approach to WLS state estimation. IEEE Trans Power Syst 26(3):1724-1732

[12] Gomez-Exposito A, Gomez-Quiles C, de la Villa Jaen A (2012) Bilinear power system state estimation. IEEE Trans Power Syst 27(1):493-501

[13] Chen YB, Ma J (2014) A mixed-integer linear programming approach for robust state estimation. J Mod Power Syst Clean Energy 2(4):366-373. doi:10.1007/s40565-014-0078-7

[14] Gol M, Abur A (2014) A robust PMU based three-phase state estimator using modal decoupling. IEEE Trans Power Syst 29(5):2292-2299

[15] Phadke AG, Thorp JS, Adamiak MG (1983) A new measurement technique for tracking voltage phasors, local system frequency, and rate of change of frequency. IEEE Trans Power Appar Syst 102(5):1025-1038

[16] Martin KE (1992) Phasor measurement system test. In: Proceedings of the British Ports Association (BPA) 1992 engineering symposium, vol 2, Portland, 31 Mar-1 Apr 1992, pp 689-704

[17] Martin KE (1999) Interutility PMU system testing. CEC document P600-00-19, California Energy Commission, Sacramento

[18] Martin KE (2002) Phasor measurements at the bonneville power administration. In: Proceedings of the 2002 conference on power systems and communications infrastructures for the future, Beijing, 23-27 Sept 2002

[19] Martin KE (2006) Phasor measurement systems in the WECC. In: Proceedings of the 2006 IEEE PES power systems conference and exposition, Atlanta, 29 Oct-1 Nov 2006, pp 132-138

[20] IEEE Std C37.118.2 (2001) IEEE standard for synchrophasor measurements for power systems

[21] Phadke AG, Thorp JS, Nuqui RF et al (2009) Recent developments in state estimation with phasor measurements. In: Proceedings of the 2009 IEEE PES power systems conference and exposition, Seattle, 15-18 Mar 2009, 7 pp

[22] Phadke AG (2010) Synchrophasor based tracking three phase state estimator and it's applications. DOE 2010 Transmission Reliability Program Peer Review, Virginia Tech, Blacksburg

[23] Jones KD, Thorp JS, Gardner RM (2013) Three-phase linear state estimation using phasor measurements. In: Proceedings of the 2013 IEEE Power and Energy Society general meeting, Vancouver, 21-25 Jul 2013, 5 pp

[24] Yang T, Sun HB, Bose A (2011) Transition to a two-level linear state estimator-part I: architecture. IEEE Trans Power Syst 26(1):46-53

[25] Yang T, Sun HB, Bose A (2011) Transition to a two-level linear state estimator-part II: algorithm. IEEE Trans Power Syst 26(1):54-62

[26] Madani V, Meliopoulos S (2014) PG\&E improves information visibility. Transm Distrib World 66(6):50-56

[27] Zhang L (2014) Validation, testing and implementation of the linear state estimator in a real power system. $\mathrm{PhD}$ Thesis, Washington State University, Pullman

[28] Zhang L, Bose A, Jampala A et al (2016) Design, testing, and implementation of a linear state estimator in a real power system. IEEE Trans Smart Grid. doi:10.1109/TSG.2015.2508283

[29] US-Canada Power System Outage Task Force (2004) Blackout 2003: final report on the August 14, 2003 blackout in the United States and Canada: causes and recommendations. Office of Electricity Delivery \& Energy Reliability, Washington, DC 
[30] Arizona-Southern California outages on September 8, 2011: causes and recommendations. FERC and NERC, Washington, 2012

[31] Report of the Enquiry Committee on grid disturbance in northern region on 30th July 2012 and in northern, eastern \& northeastern region on 31st July 2012. The Enquiry Committee, Ministry of Commerce and Industry, Government of India, New Delhi, 2012

[32] Synchrophasor analytics-documentation (2014) CodePlex: Open Source Project Hostin

[33] Common information model (CIM) (2009) European Network of Transmission System Operators for Electricity (ENTSO-E), Brussels, Belgium

[34] Chakhchoukh Y, Vittal V, Heydt GT (2014) PMU based state estimation by integrating correlation. IEEE Trans Power Syst 29(2):617-626

[35] Grainger JJ, Stevenson WD (2003) Power system analysis. McGraw-Hill, New York, pp 655-664

[36] Phadke AG, Thorp JS (2008) Synchronized phasor measurements and their applications. Springer, Boston, pp 150-163

[37] Patel M, Aivolaitas S, Allen E et al (2010) Real-time application of synchrophasors for improving reliability. North American Electric Reliability Corporation (NERC), Princeton

[38] Chen H, Mo JZ, Kothapa U et al (2014) Development of an online synchrophasor wide area dynamics monitoring platform. In: Proceedings of the 2014 IEEE PES transmission \& distribution conference and exposition, Chicago, 14-17 Apr 2014, 5 pp

[39] Chen H, Martin KE, Bhargava B et al (2014) On-line islanding detection application in the real-time dynamics monitoring system (RTDMS). In: Proceedings of the 2014 IEEE PES transmission \& distribution conference and exposition, Chicago, 14-17 Apr 2014, 4 pp

Heng CHEN received his B.S. degree in power systems and its automation from North China Electric Power University, Baoding, China, in 2009, and M.S. degree in electrical engineering from the University of Wisconsin-Madison, Madison, USA, in 2011. He is currently a Sr. power systems engineer at Electric Power Group,
Pasadena, USA. He is engaged mainly in research, design and implementation on applications of synchrophasor technology, and utilization of synchrophasor data for grid performance analysis and real-time wide area monitoring. He is a licensed Professional Engineer in Electrical Engineering in the State of California, USA.

Lin ZHANG received his B.Eng. degree from Huazhong University of Science and Technology, Wuhan, China, and M.S degree from Wuhan University, Wuhan, China, and Ph.D degree from Washington State University, Pullman, USA. His special fields of interest include power system real-time modeling and linear state estimation using synchrophasor. He is now a power systems engineer at Electric Power Group, Pasadena, USA. His research interests include synchrophasor technologies, synchrophasor data quality and linear state estimator.

Jianzhong MO received his Ph.D degree from Shanghai Jiaotong University, Shanghai, China, in 1998. His research interests include distributed system, large volume real-time data analytics, data centric visualization, synchrophasor technology, wide area measurement system, and their applications in power industry. He is now the chief system architect at Electric Power Group, Pasadena, USA. Before joining Electric Power Group, he was a post-doctoral researcher at the University of Wisconsin-Madison, Madison, USA.

Kenneth E. MARTIN is a Sr. Principal Engineer at Electric Power Group. He has over 35 years' experience in the electric utility industry, first at the Bonneville Power Administration (BPA) in communication, precise timing, instrumentation, and testing. He started working with synchrophasor measurement with the original PMUs in 1987 and conducted the first PMU tests. He developed the phasor measurement system at BPA including building the first phasor data concentrator, and supported similar developments at many utilities. He chaired the development of the IEEE C37.118 Synchrophasor Standards from the 2005 original, to the 2011 updates, and the 2014 amendment. He was a lead for the IEC 61850, part 90-5 and is the convener for IEC 60255-118-1 developing the joint IECIEEE measurement standard. He is a Fellow of IEEE and a registered Professional Engineer in the State of Oregon, USA. 